\title{
REQUERIMIENTOS HÍDRICOS DEL PLÁTANO DOMINICO- HARTÓN (Musa AAB SIMMONDS) EN LA REGIÓN SANTÁGUEDA (PALESTINA, CALDAS)
}

\section{HYDRIC REQUIREMENTS OF PLANTAIN DOMINICO-HARTON (Musa AAB SIMMONDS) INTHE SANTAGUEDA REGION (PALESTINA, CALDAS)}

\author{
Ángela María Castaño ${ }^{1}$, Manuel Aristizábal $^{2}$, Héctor González ${ }^{3}$ \\ ${ }^{1}$ I.A. Programa Agronomía. Universidad de Caldas. angelacpineda@hotmail.com; ${ }^{2}$ I.A. M.Sc. Profesor catedrático. Maestría \\ Fitopatología. Universidad de Caldas. hector.gonzalez_o@ucalas.edu.co; ${ }^{3}$ I.A. M.Sc. Profesor Titular. Programa Agronomía. \\ Universidad de Caldas. Calle 65 No. 26-10. Manizales, Caldas. manuel.aristizabal@ucaldas.edu.co
}

Rev. U.D.C.A Act. \& Div. Cient. 15(2): 331 - 338, 2012

RESUMEN

El presente estudio, se desarrolló en la granja Montelindo, de la Universidad de Caldas, con el fin de establecer los requerimientos hídricos del cultivo del plátano, con base en el cálculo de la evapotranspiración de referencia, calculada por el método de Penman-Monteith y la evapotranspiración del cultivo, según el estado fenológico de la planta. Se sembraron plantas de plátano Dominico-Hartón, a $2 \mathrm{~m}$ entre plantas y $3 \mathrm{~m}$, entre surcos y, oportunamente, se efectuaron las prácticas agronómicas necesarias. Al inicio del estudio, se determinaron la capacidad de campo, el punto de marchitez permanente y la lámina de agua aprovechable. Semanalmente, se hicieron registros de los elementos climáticos, el crecimiento de las plantas y la tensión de humedad del suelo, utilizando tensiómetros ubicados a $30,48 \mathrm{~cm}$ y $45,72 \mathrm{~cm}$ de profundidad. Se estableció que en la región Santágueda, las plantas de Dominico-Hartón, bajo condiciones adecuadas de cultivo, requieren $1.141 \mathrm{~mm}$ de agua, durante un ciclo del cultivo; que el consumo de agua aumenta gradualmente a medida que el cultivo avanza en su desarrollo, siendo la floración la época de mayor uso de agua y la más crítica, en cuanto al déficit hídrico. También, se encontró que la altura de la planta, la emisión de hojas y el diámetro del pseudotallo tienen relación directa con la evapotranspiración del cultivo.

Palabras clave: Evapotranspiración, consumo de agua, crecimiento de la planta.

\section{SUMMARY}

The present study was developed at the farm 'Montelindo' of the University of Caldas in order to establish the hydric requirements of the plantain crop, based on the evapotranspiration calculated by Penman-Monteith method and the crop evapotranspiration according to the phonological stage of the plant. Plants of Dominico-Harton plantain were planted at $2 \mathrm{~m}$ between plants and $3 \mathrm{~m}$ between rows and the necessary agronomic practices were applied on time. At the beginning of the study the field capacity, the permanent wilting point and the usable water table were determined. Weekly, registers of climatic elements, plants growth and soil humidity tension with tensiometers located at 40,48 and 45, 72 inches deep, were made. It was established that at the Santagueda region the Dominico-Harton plants, under appropriated culture conditions, require $1.141 \mathrm{~mm}$ of water during a culture cycle; that water consumption increases as the development of the crop advances, being the flowering period the time of highest water consumption and also the most critical one in relation to water deficit. It was also found that plant height, leaves emission and pseudostem diameter have a direct relation with crop evapotranspiration.

Key words: Evapotranspiration, water consumption, plant growth.

\section{INTRODUCCIÓN}

El cultivo de plátano en Colombia es uno de los más importantes, por ser un producto básico en la alimentación y por generar empleo e ingresos para el país. En la zona cafetera central mantiene su posición, después del café, 
siendo el Dominico-Hartón la culti-variedad más utilizada, por su adaptación al clima y por su preferencia en los mercados. Es importante identificar las interacciones entre la planta y el ambiente, especialmente, para establecer el potencial productivo de una zona o, simplemente, para optimizar el uso de los recursos, como el agua. Para este fin es necesario conocer las necesidades hídricas del cultivo (Morillo, 1999), que se definen como la cantidad de agua requerida para compensar la pérdida por evapotranspiración (Allen et al. 2006), que esta determinada por el clima, las características del cultivo y el manejo agronómico (Jaramillo, 2005).

El plátano, por la morfología de la planta e hidratación de sus tejidos, requiere abundante cantidad de agua disponible en el suelo, para que el crecimiento y el desarrollo ocurran normalmente (Belalcázar, 1991), ya que sus necesidades hídricas se deben a que tiene gran superficie foliar transpirante, siendo más exigente en agua que otras especies (Martínez, 1983). El plátano es sensible a la falta de agua durante todo su ciclo de vida, pero, particularmente, durante la primera parte del período vegetativo, así como durante la floración y la formación del racimo (Doorenbos \& Kassam, 1980). No hay suficiente información experimental que muestre si bajo la distribución de las lluvias en la región Santáqueda, el plátano experimenta situaciones de déficit y de exceso de agua para su desarrollo.

Según Turner et al. (2007) no se sabe claramente la relación entre el consumo de agua y la productividad del banano; por su parte, Mahouachi (2008) estableció que el fruto ajusta su tasa de crecimiento al suministro de agua aprovechable por la planta; no obstante, Van Vosselen et al. (2005), al comparar dos métodos para calcular el consumo de agua por la planta de banano, establecieron que el modelo de simulación Suelo-Agua-Planta-Atmósfera tiende a subestimar la evapotranspiración real, además que requiere gran cantidad de datos; mientras que el método del balance de agua sigue siendo una opción útil para dicho propósito y requiere poca información. Por tales razones, se llevó a cabo el presente estudio, con el objetivo de calcular los requerimientos hídricos del plátano Dominico Hartón, determinando la Evapotranspiración de Referencia (ETo), por el método de Penman-Monteith y la Evapotranspiración del Cultivo (ETc), según el estado fenológico de la planta.

\section{MATERIALES Y MÉTODOS}

El estudio, se llevó a cabo en la granja Montelindo, de la Universidad de Caldas, ubicada en Palestina (Caldas), a $5^{\circ}$ $4^{\prime} \mathrm{N}, 75^{\circ} 40^{\prime} \mathrm{W}, 1026 \mathrm{msnm}$, temperatura media de $22,5^{\circ} \mathrm{C}$, máxima de $29,7^{\circ} \mathrm{C}$ y mínima de $17,6{ }^{\circ} \mathrm{C}$, humedad relativa del $76 \%$, precipitación anual de $2100 \mathrm{~mm}$, brillo solar de 1996 horas/año y suelos de origen volcánico, Typic Dystrandept.
Para este estudio, se sembraron 160 plantas, con distancia de 2,0 m entre plantas y $3 \mathrm{~m}$ entre surcos. A partir de las 8 semanas, después de siembra, se comenzó la medición semanal de las variables de crecimiento en 20 plantas: altura de la planta (AP), número de hojas emitidas (NHE), número de hojas funcionales (NHF) y área foliar en $\mathrm{m}^{2}$ (AF), calculada mediante la fórmula de Martínez (1984):

$$
\text { Área foliar (AF) }=\text { Largo } \mathrm{x} \text { ancho } \mathrm{x} \text { 0,8 }
$$

El diámetro del pseudotallo en cm (DP), se comenzó a medir a partir de 15 semanas, después de siembra, cuando la mayoría de las plantas alcanzaron $1 \mathrm{~m}$ de altura.

Para medir la tensión de humedad en el suelo a las 7 semanas, después de siembra, se instalaron en el lote cuatro estaciones de tensiómetros marca Irrometer ${ }^{\circledR}$, cada una con dos tensiómetros, uno de $12 "(30,48 \mathrm{~cm})$ y otro de 18 " $(45,72 \mathrm{~cm})$, en el área radical activa $(\mathrm{a} 20 \mathrm{~cm}$ de la base del pseudotallo) y, semanalmente, se registraron los datos de la tensión de humedad del suelo en centibares.

La humedad en capacidad de campo, se determinó utilizando el método de campo, recomendado por Cenicaña (2004), que consistió en construir una piscina de $1 \mathrm{~m}^{2}$ en el área más representativa del lote de estudio, antes de la siembra. Los bordes de la piscina, se construyeron para conformar un dique de unos $20 \mathrm{~cm}$ de altura y, luego, se aplicó una lámina de agua de $100 \mathrm{~mm}$ o $100 \mathrm{Lm}^{-2}$ y se cubrió con plástico oscuro, para evitar la evaporación. Después de $48 \mathrm{~h}$, se tomaron cuatro muestras de suelo de $200 \mathrm{~g} \mathrm{a}$ $30 \mathrm{~cm}$ de profundidad, se empacaron herméticamente y se llevaron al laboratorio de suelos de la Universidad de Caldas, donde fueron secadas en un horno a $125^{\circ} \mathrm{C}$ por $24 \mathrm{~h}$, para obtener el contenido gravimétrico de humedad a capacidad de campo.

La humedad gravimétrica, se obtuvo a partir de la siguiente expresión:

$$
\mathrm{W}=\frac{\text { Psh }- \text { Pss }}{\text { Pss }} \times 100
$$

Donde:

W: Humedad gravimétrica (\%)

Psh: Peso del suelo húmedo $(\mathrm{g})$

Pss: Peso del suelo seco $(\mathrm{g})$

La humedad en punto de marchitez permanente, se estimó aplicando el método biológico, recomendado por Cenicaña (2004), para lo cual, se utilizaron cuatro recipientes de poliestireno, con capacidad de $500 \mathrm{~g}$ de suelo. Se recolectaron muestras de suelo del horizonte A y, en cada recipiente, se sembraron tres semillas de girasol enano (Helianthus sp.), 
que fueron mantenidas con riego constante. Cuando las plantas alcanzaron una altura de $10 \mathrm{~cm}$, se dejó una planta por recipiente y se suspendió el suministro de agua, sellando con plástico la parte superior de los recipientes. Se comenzó la observación semanal del vigor de las plantas; a las dos semanas mostraron síntomas de sequía y pérdida de turgencia, alcanzado el punto de marchitez permanente. En ese momento, las muestras de suelo de los recipientes fueron llevadas al laboratorio de biotecnología de la Universidad de Caldas, donde fueron secadas en un horno a $125^{\circ} \mathrm{C}$ por $24 \mathrm{~h}$, para obtener el contenido gravimétrico de humedad, a punto de marchitez permanente.

El contenido de humedad del suelo como lámina de agua en $\mathrm{mm}$, se calculó mediante la siguiente ecuación:

$$
\mathrm{L}=\frac{\mathrm{W} \times \mathrm{Da}}{100} \times \operatorname{Pr}
$$

Donde:

L: Lámina de agua en $\mathrm{mm}$

W: Humedad gravimétrica (\%)

Pr: Profundidad radicular $(\mathrm{cm})$

Da: Densidad aparente $\left(\mathrm{g} / \mathrm{cm}^{3}\right)$

Para calcular la Da en campo, se utilizaron cilindros de volumen conocido, donde se recolectaron muestras de suelo del horizonte $\mathrm{A}$, que se secaron en horno a $105^{\circ} \mathrm{C}$ por $24 \mathrm{~h}$, para relacionar el peso seco del suelo con el volumen total del cilindro, cuya expresión es:

$$
\begin{aligned}
& \mathrm{Da}=\frac{\mathrm{Pss}}{\mathrm{Vt}} \\
& \mathrm{Vt}=\pi \mathrm{R}^{2} \mathrm{~h}
\end{aligned}
$$

Donde:

Da: Densidad aparente $\left(\mathrm{g} / \mathrm{cm}^{3}\right)$

Pss: Peso del suelo seco $(\mathrm{g})$

Vt: Volumen total, siendo $\mathrm{R}$ el radio de la base y h la altura del cilindro.

Lámina de agua aprovechable, se determinó utilizando la siguiente expresión:

$$
\mathrm{LAA}=\frac{\mathrm{CC}-\mathrm{PMP}}{100} \times \mathrm{Da} \times \operatorname{Pr} \times 10
$$

Donde:

LAA: Lámina de agua aprovechable (mm)

CC: Capacidad de campo (\% de humedad)

PMP: Punto de marchitez permanente (\% de humedad)

Da: Densidad aparente $\left(\mathrm{g} / \mathrm{cm}^{3}\right)$

Pr: Profundidad radicular $(\mathrm{cm})$
Los datos climáticos diarios de temperaturas media, máxima y mínima $\left({ }^{\circ} \mathrm{C}\right)$, humedad relativa $(\%)$, precipitación $(\mathrm{mm})$ y brillo solar (horas), para el período comprendido entre marzo de 2008 y mayo de 2009, fueron obtenidos del Centro Nacional de Investigaciones del Café (CENICAFÉ), los cuales, habían sido registrados en la estación Santágueda, ubicada en la granja Montelindo.

Los requerimientos hídricos del cultivo, se establecieron mediante el cálculo de la evapotranspiración de referencia (ETo en $\mathrm{mm}$ ), para lo cual, se utilizó el método de PenmanMonteith (Allen et al. 2006):

$$
\lambda E T=\frac{\Delta R_{n}+\rho C p \frac{\left(e_{s}-e_{r}\right)}{r_{a}}}{\Delta+\gamma\left(1+r_{s} / r_{a}\right)}
$$

Donde:

$\lambda$ ET: Evapotranspiración de referencia, $\mathrm{mm}$

$\lambda$ : Calor latente de vaporización de agua $(\mathrm{J} / \mathrm{kg})$

D: Pendiente de la curva de saturación de vapor de presión, (mbar/K)

$\mathrm{R}_{\mathrm{n}}$ : Radiación solar neta, $\left(\mathrm{W} / \mathrm{m}^{2}\right)$

$\rho$ : Densidad del aire, $\left(\mathrm{kg} / \mathrm{m}^{3}\right)$

$\mathrm{Cp}=$ Calor especifico del aire a presión constante, $(\mathrm{J} / \mathrm{kg} . \mathrm{K})$

$\mathrm{e}_{\mathrm{s}}=$ Presión de vapor a punto de saturación, (mbar)

$\mathrm{e}_{\mathrm{r}}=$ Presión de vapor real, (mbar)

$\mathrm{r}_{\mathrm{a}}=$ Resistencia aerodinámica, $(\mathrm{s} / \mathrm{m})$

$\gamma=$ Coeficiente psicrométrico, $(\mathrm{mbar} / \mathrm{K})$

$\mathrm{r}_{\mathrm{s}}=$ Resistencia superficial, $(\mathrm{s} / \mathrm{m})$

Para cuantificar la evapotranspiración del cultivo (ETc en $\mathrm{mm}$ ), se emplearon la fórmula y los coeficientes del cultivo (Kc), determinados por Doorenbos \& Pruitt (1977), teniendo en cuenta la etapa de desarrollo del cultivo:

$$
\mathrm{ETc}=\mathrm{ETo} \times \mathrm{Kc}
$$

Donde:

ETc: Evapotranspiración del cultivo en mm

ETo: Evapotranspiración de referencia en mm

Kc: Coeficiente del cultivo

\section{RESULTADOS Y DISCUSIÓN}

La duración total del ciclo del cultivo fue de 60 semanas, la temperatura media de $23^{\circ} \mathrm{C}$, la máxima de $28,8^{\circ} \mathrm{C}$ y la mínima de $18,6^{\circ} \mathrm{C}$, encontrándose entre los rangos adecuados para Dominico-Hartón, establecidos por Belalcázar (1991). La humedad relativa fue del $78,8 \%$ y el brillo solar acumulado fue de 2.049,3 horas. La precipitación acumulada alcanzó $3.933,1 \mathrm{~mm}$, durante el ciclo del cultivo.

La densidad aparente del suelo fue de $0,866 \mathrm{~g} \mathrm{~cm}^{-3}$, con la cual, se calculó una humedad gravimétrica de $35,4 \%$, para 
la capacidad de campo (CC) y de $17,9 \%$, para el punto de marchitez permanente (PMP). La lámina de agua obtenida fue de 91,97mm, para CC, que se aplicó en el balance hídrico, como capacidad de almacenamiento del suelo y de 46,48mm, para PMP, con una lámina de agua de $46,45 \mathrm{~mm}$.

La tensión de humedad del suelo a dos profundidades diferentes fue similar a través del tiempo, manteniendo los niveles de humedad entre suelo saturado y capacidad de campo, excepto en las semanas 25 a 30 del cultivo, en las que hubo un déficit de agua considerable, que coincidió con la etapa de iniciación floral, lo que aumentó la tensión de humedad del suelo, indicando el intervalo de riego. La tensión de humedad, se mantuvo alrededor de 9,3cb, para las dos profundidades evaluadas, que según los rangos de humedad de los tensiómetros corresponde a suelo saturado (Figura 1); a este respecto, Orozco \& Pérez (2006) mencionan que las raíces del plátano extraen agua del suelo únicamente a valores bajos de succión, por lo cual, la humedad del suelo se debe mantener cerca de la capacidad de campo, para evitar estrés por agua. Además, el hecho que las raíces no tengan aparentemente un poder de succión importante, sugiere que el suelo deberá estar siempre con un nivel alto de humedad (Santacruz \& Santacruz, 1995).

Las tensiones más altas, se presentaron a $23,7 \mathrm{cb}$, para los tensiómetros de 12 " y a $24,7 \mathrm{cb}$, para los de 18 ", ambos valores en la semana 27. A lo largo del ciclo del cultivo, los mayores valores de tensión de humedad se dieron a partir de la semana 39, para los tensiómetros de menor profundidad, con un promedio de $13,5 \mathrm{cb}$, ubicándose en el rango de capacidad de campo, en comparación con valores menores a mayor profundidad, alcanzando 7,9cb y suelo saturado, para el mismo período (Figura 1), indicando mayor tensión a menor profundidad, debido a que la mayoría de las raíces se desarrollan en los primeros 20 a $40 \mathrm{~cm}$ de la superficie del suelo, con una distribución espacial de carácter radial horizontal (Belalcázar, 1991). Este comportamiento se debe también al mayor consumo de agua por la planta, a partir de la iniciación floral y el desarrollo de la bellota, dado que cuenta con una extensa área foliar, que ocasiona un aumento en la transpiración y requiere más agua, durante la floración y el llenado de frutos (Doorenbos \& Pruitt, 1977).

En las primeras etapas del cultivo, la mayor tensión de humedad se presenta en los tensiómetros a mayor profundidad, con 9,6cb. A partir de la semana 29 , cuando el cultivo se encuentra en la etapa de diferenciación floral, la tendencia cambia y la tensión es mayor en los tensiómetros de menor profundidad, con 11,5cb (Figura 1), debido a que la evaporación y la transpiración ocurren simultáneamente y no hay manera sencilla de separarlas. En las primeras etapas del cultivo, el agua se pierde, principalmente, por evaporación directa del suelo, pero con el desarrollo del cultivo y cuando este cubre totalmente el suelo, la transpiración se convierte en el proceso principal. En el momento de la siembra, casi el $100 \%$ de la evapotranspiración ocurre en forma de evaporación, mientras que cuando la cobertura vegetal es completa, más del $90 \%$ de la ET ocurre como transpiración (Allen et al. 2006). En la etapa reproductiva del cultivo, la tensión de humedad del suelo es más alta a menor profundidad, por la presencia de un mayor porcentaje de raíces en esta zona y por la transpiración que es mayor, por presentar una superficie foliar más extensa en esta etapa (Aristizábal, 2008).

La evapotranspiración de referencia (ETo) siempre fue mayor que la evapotranspiración del cultivo (ETc) en los

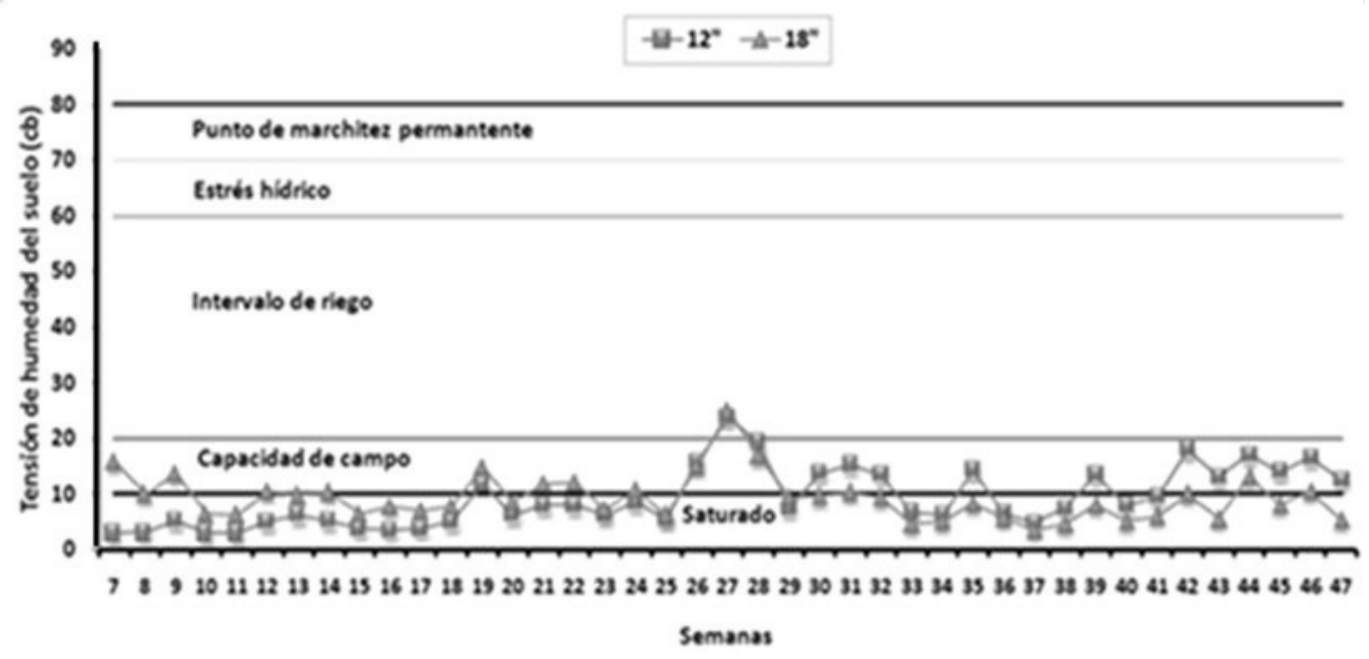

Figura 1. Tensión de humedad del suelo a $12 "(30,48 \mathrm{~cm})$ y $18 ”(45,72 \mathrm{~cm})$. 
dos períodos en que fueron calculadas (Tabla 1). A partir de la primera, se estableció que en la región Santágueda las plantas de plátano Dominico Hartón, bajo condiciones óptimas de cultivo y una densidad de 1.666 plantas ha $^{-1}$, se requieren 1.140,6 $\mathrm{mm}$ de agua en un ciclo total de cultivo, que corresponden al $72 \%$ de la evapotranspiración real. En estudios realizados por diversos autores, las necesidades hídricas del plátano, para la zona tropical, varían entre 1.200 y $2.314 \mathrm{~mm} / \mathrm{año}$, con un promedio de $1.756 \mathrm{~mm} /$ año (Peña, 2005; Doorenbos \& Kassam, 1980; Sastry, 1988; Santacruz \& Santacruz, 1995). En la práctica, se requieren alrededor de $150 \mathrm{~mm}$ mensuales de precipitación $\left(1,500 \mathrm{~m}^{3} \mathrm{ha}^{-1}\right)$ para satisfacer las necesidades hídricas del plátano (Cayón, 2004). En este caso, la cantidad de agua requerida por el cultivo es menor que la reportada en la literatura; pero el estudio in situ determinó que la región Santágueda presenta las condiciones hídricas adecuadas para el establecimiento exitoso del cultivo del plátano, porque las precipitaciones anuales son suficientes para cubrir los requerimientos hídricos de la culti-variedad Dominico-Hartón, a pesar que, como lo mencionan Doorenbos \& Kassam (1980), por su gran superficie foliar y pseudotallos carnosos, los requerimientos de agua son elevados, en comparación con otras especies. Peña (2005) y Santacruz \& Santacruz (1995) señalan que las reservas de agua de estas plantas son mínimas y pueden ser gastadas en algunas horas de transpiración normal, lo cual, las obliga a equilibrar constantemente las pérdidas de agua. Por esto, cuando haya distribución anormal o escasez de la precipitación y se presenten valores inferiores a los requeridos en cada etapa del cultivo, es necesario tomar medidas para evitar el estrés hídrico, como la utilización de coberturas que reducen la evaporación y conservan la humedad en el suelo. De acuerdo con Jaramillo (2005), la mejor economía del agua en regiones secas o con períodos de deficiencia de agua acentuados o disminución significativa de las lluvias, como cuando se presenta el fenómeno de El Niño, se logra protegiendo el suelo con cobertura muerta, la cual, se puede obtener cortando las arvenses al inicio del período seco o, como en el caso del plátano, dejando en el lote los residuos generados por las labores de control fitosanitario, como el deshoje, el despunte y el desguasque.

El consumo de agua por la planta aumenta a medida que crece. En las etapas de plántula (V1) y formación de hijuelos (V2), durante las primeras ocho semanas del cultivo, los requerimientos hídricos son constantes, alrededor de $11,3 \mathrm{~mm}$ semanales. Durante el alargamiento inicial de entrenudos (V3), se mantiene un consumo de 10,4mm; pero a partir de la semana 18 , después de siembra, el consumo de agua aumenta a $13,1 \mathrm{~mm}$ por semana. Cuando la planta pasa al estado reproductivo el uso consuntivo aumenta gradualmente. En la etapa de iniciación floral (R4), el consumo promedio fue de $20,8 \mathrm{~mm}$ semanales y en la etapa R5 (desarrollo de la bellota), el promedio fue de $27,3 \mathrm{~mm}$, por semana; alcanzando los consumos más altos durante las semanas 34 a 45 , con un promedio de $26,5 \mathrm{~mm}$ semanales, siendo la floración la época más limitante, en cuanto al déficit hídrico. Este comportamiento es similar al reportado por Peña (2005), que considera el período inflorescencia-floración-desarrollo del fruto el más exigente en cuanto al consumo de agua. A partir de la semana 46, el consumo de agua disminuye levemente, con un consumo semanal promedio de $24,3 \mathrm{~mm}$; sin embargo, en la etapa de llenado del racimo, la planta requiere $20 \mathrm{~mm}$ semanales en promedio. Finalmente, si se deja más tiempo el racimo en la planta y se produce allí la maduración, el consumo de agua es de $22,8 \mathrm{~mm}$ semanales. El requerimiento más bajo de agua, se presentó en la semana nueve, con $8,79 \mathrm{~mm}$ y el más alto fue en la semana 41, con 29,4mm (Figura 2).

Las etapas que más agua requieren son: iniciación floral (R4), desarrollo de la bellota (R5), floración (R6) e inicio de la formación del racimo (R7). Según Belalcázar (1991) estas etapas tienen de singular importancia, porque de su correcta evolución va a depender el rendimiento del cultivo. En el período de siembra a belloteo el uso consuntivo del cultivo

Tabla 1. Evapotranspiración de referencia por Penman-Monteith (ETo en mm) y Evapotranspiración del Cultivo (ETc en mm), para plátano Dominico Hartón.

\begin{tabular}{|c|c|c|c|}
\hline Período & $\begin{array}{c}\text { Tiempo } \\
\text { (Semanas) }\end{array}$ & $\begin{array}{c}\text { ETo Penman } \\
\text { Monteith }(\mathrm{mm})\end{array}$ & $\begin{array}{c}\text { ETc } \\
(\mathrm{mm})\end{array}$ \\
\hline S-B* & 39 & 1051,4 & 640,5 \\
\hline B-C & 21 & 541,7 & 500,1 \\
\hline TOTAL & 60 & 1593,1 & 1140,6 \\
\hline
\end{tabular}

*S-B: Siembra a Belloteo, B-C: Belloteo a cosecha 


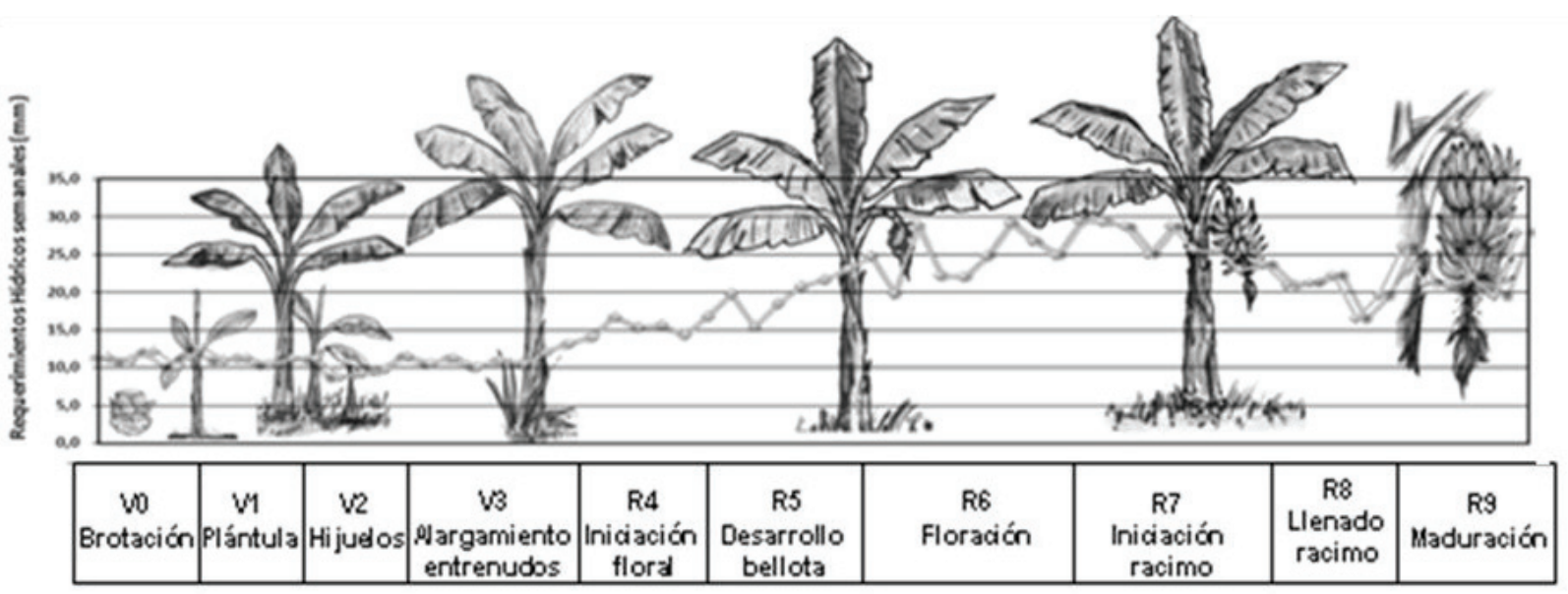

Figura 2. Requerimientos hídricos semanales de acuerdo con la etapa de crecimiento del plátano Dominico Hartón, en la región Santágueda, Palestina, Caldas. Fuente Dibujos: Belalcázar \& Rosales (2001).

fue de $640.5 \mathrm{~mm}$, disminuyendo de belloteo a cosecha, donde requirió $500,1 \mathrm{~mm}$ de agua.

El requerimiento hídrico no es constante a través del tiempo, ya que dependiendo de la etapa de desarrollo de la planta se requieren cantidades diferentes de agua, presentando un aumento progresivo, a medida que las plantas avanzan en su crecimiento, siendo la floración la época de mayor consumo de agua y, por tanto, la más limitante, en cuanto al déficit hídrico (Figura 2). Este comportamiento se debe a que con el transcurrir del tiempo las plantas aumentan su área foliar (Aristizábal, 2008) y el número de hijuelos, cuya formación se inicia, aproximadamente, a los tres meses después de la siembra (Aristizábal \& Jaramillo, 2010), lo cual, determina que el sistema de raíces sea más abundante. Nótese que después de la floración, cuando comienza la iniciación del racimo, se presenta una caída en el requerimiento de agua (Figura 2), lo cual, se debe a que para esa época la emisión de hojas ha cesado y esto se traduce en disminución del área foliar (Aristizábal, 2008) y, en consecuencia, en menor transpiración.

Se estableció que existe una correlación positiva y altamente significativa $(\mathrm{P}<0,001)$ entre la evapotranspiración del cultivo, interpretada como el consumo de agua (Turner et al. 2007), con todos los parámetros de crecimiento, es decir, a medida que la planta aumenta en su desarrollo, la cantidad de agua que consume también es mayor. Además, existe una correlación positiva y altamente significativa entre las variables de crecimiento, con excepción del área foliar y el número de hojas funcionales, que no mostraron asociación entre ellas, posiblemente, por la influencia de enfermedades, como las Sigatokas negra y amarilla (Mycosphaerella fijiensis y M. musicola), que afectan de manera directa el área foliar, ya que al realizar las prácticas culturales necesarias, para su manejo, se reduce el número de hojas funcionales.

Las relaciones directas y altamente significativas y confiables de la altura de la planta (Figura 3A), el diámetro del pseudotallo (Figura 3B) y el número de hojas emitidas (Figura 3C) con la ETc, indican la importancia del suministro del agua, para garantizar un crecimiento normal del cultivo, con mayor efecto en la altura de la panta y en la producción de hojas que en el grosor del pseudotallo. La tasa de emisión foliar está estrechamente relacionada con las condiciones ambientales. De acuerdo con Belalcázar (1991), uno de los factores que más influye sobre la emergencia de las hojas son los períodos largos de sequía, que pueden incrementar el proceso de emisión foliar en 15 días o más.

En el caso del AF y la ETc, el bajo coeficiente de determinación indica que el porcentaje de variabilidad restante se explica porque los procesos de evaporación y de transpiración no son diferenciables, correspondiendo a la combinación del agua perdida por evaporación desde el suelo y la absorbida por las raíces que, posteriormente, se pierde en su totalidad, a través de la superficie de la hojas, porque la mayor parte del agua absorbida es liberada a la atmósfera en forma de vapor y sólo entre $1 \%$ y $2 \%$ es utilizada para la formación de los tejidos (Aristizábal, 2003). La relación directa entre la altura de la planta y la ETc y el alto coeficiente de determinación para dicha asociación (Figura 3A) indican la influencia que el suministro de agua tiene en el crecimiento de la planta, hasta el punto que por cada mililitro de evapotranspiración, la altura de la planta aumentaría en $12 \mathrm{~cm}$.

Los resultados obtenidos y el análisis de los mismos permiten inferir que: 

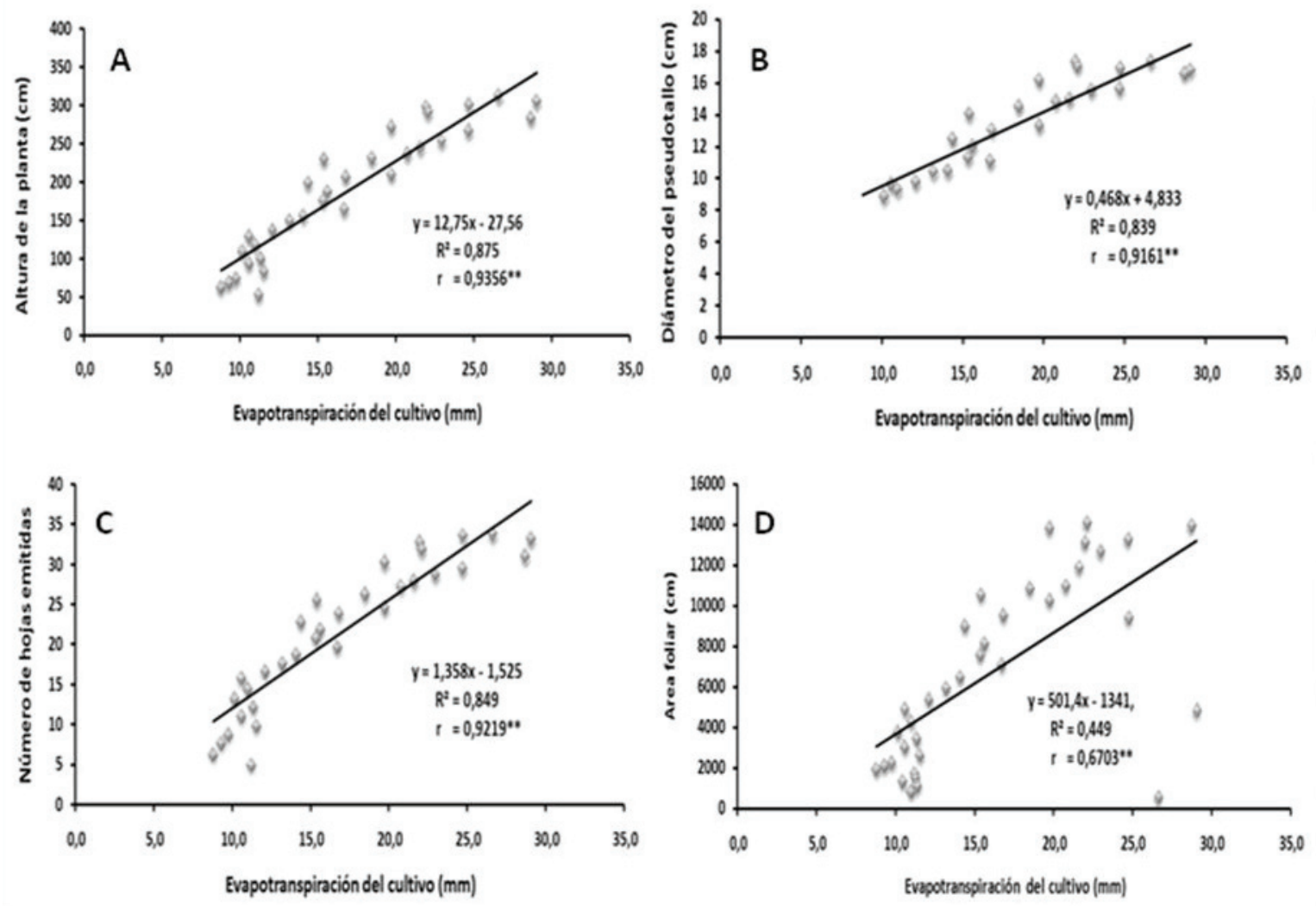

Figura 3. Altura de la planta (A), diámetro del pseudotallo evapotranspiración del cultivo para plátano Dominico Hartón.

(B), hojas emitidas (C) y área foliar (D) en función de la

Las condiciones climáticas en la región Santágueda se encuentran en los rangos adecuados para un óptimo desarrollo del plátano Dominico-Hartón; sin embargo, se pueden presentar épocas de déficit o exceso de agua, indicando que la distribución de la precipitación, en ocasiones, no coincide con las etapas fenológicas del cultivo.

- Los valores de lluvia presentes durante el ciclo del cultivo en la región son suficientes para satisfacer los requerimientos hídricos del plátano Dominico-Hartón y cuando su distribución es normal, se logra mantener los niveles de agua en la cantidad requerida por las plantas.

- En suelos francos, como los de la región Santágueda, la mayoría del tiempo y los niveles de humedad se mantienen cercanos a la Capacidad de Campo y a Saturación, garantizando el abastecimiento constante de agua para el cultivo.

- Los requerimientos hídricos del cultivo no son constantes a través del tiempo, dependiendo de la etapa de desarrollo, las plantas requieren cantidades diferentes de agua, presentando un aumento progresivo a medida época de mayor consumo de agua y, por tanto, la más limitante, en cuanto al déficit hídrico.

Agradecimientos: Al Programa Plátano de la Universidad de Caldas por el apoyo financiero para la realización del trabajo de campo. Conflicto de intereses: El manuscrito fue preparado y revisado con la participación de todos los autores, quienes declaramos que no existe ningún conflicto de intereses que ponga en riesgo la validez de los resultados presentados.

\section{BIBLIOGRAFÍA}

1. ALLEN, R.; PEREIRA, L.; RAES, D.; SMITH, M. 2006. Evapotranspiración del cultivo, Guías para la determinación de los requerimientos de agua de los cultivos. FAO (Italia). 298p.

2. ARISTIZÁBAL, L.M. 2003. Fisiología Vegetal. Universidad de Caldas. Artes Gráficas Tizan (Colombia). 306p. 
3. ARISTIZÁBAL, L.M. 2008. Evaluación del crecimiento y desarrollo foliar del plátano Hondureño Enano (Musa AAB) en una región cafetera de Colombia. Agronomía (Colombia). 16(2):23-30.

4. ARISTIZÁBAL, L.M.; JARAMILLO, G.C. 2010. Identificación y descripción de las etapas de crecimiento del plátano Dominico Hartón (Musa AAB). Agronomía. 18(1):29-40.

5. BELALCÁZAR, S. 1991. El Cultivo del Plátano en el trópico. Manual de Asistencia Técnica No 50. Instituto Colombiano Agropecuario ICA (Colombia). $376 p$.

6. BELALCÁZAR, S.; ROSALES, F. 2001 Siembra y explotación del cultivo del plátano. Módulos para la capacitación de productores. Red Internacional para el Mejoramiento del Banano y el Plátano INIBAP. (Francia). 171p.

7. CAYÓN, S.D.G. 2004. Ecofisiología y productividad del plátano (Musa AAB Simmonds). XVI Reunión Internacional de ACORBAT. Documento especial p.172-183.

8. CENICAÑA. 2004. Avances técnicos para la programación y el manejo del riego en caña de azúcar. Serie Técnica N. 33 (Colombia). 2a Edición. $66 \mathrm{p}$.

9. DOORENBOS, J.; KASSAM, A.H. 1980. Efectos del agua sobre el rendimiento de los cultivos. Estudio de Riego y Drenaje $N^{\circ} 33$ (Italia). Organización de las Naciones Unidades para la Agricultura y la Alimentación FAO. 193p.

10. DOORENBOS, J.; PRUITT, W.O. 1977. Las necesidades de agua de los cultivos. Estudio de Riego y Drenaje N⿳o 24 (Italia). Organización de las Naciones Unidades para la Agricultura y la Alimentación FAO. 179p.

11. JARAMILlO, A. 2005. Clima Andino y Café en Colombia. Centro Nacional de Investigaciones de Café CENICAFÉ. (Colombia). 192p.

12. MARTíNEZ, G.A. 1983. Ecología del Cultivo del Plátano. Memorias. Primer Seminario Internacional sobre el Plátano. Publicaciones Universidad de Caldas. (Colombia). p.137-143.
13. MARTÍNEZ, G.A. 1984. Determinación del área mínima foliar en plátano en el trópico húmedo. Revista ICA. 19(2):183-187.

14. MAHOUACHI, J. 2008. Growth and mineral nutrient content of developing fruits on banana plant (Musa acuminata AAA 'Gran Nain') subjected to water stress and recovery. J. Hort. Sci. Biotech. 82:839844.

15. MORILLO, R. 1999. Necesidades hídricas del cultivo de remolacha en Castilla y León. Disponible desde Internet en: http://4w.cajaduero.es/agro/public/ remolacha/Riego4.htm (con acceso 28/04/07).

16. OROZCO, J.; PÉREZ, O. 2006. Tensión de humedad del suelo y fertilización nitrogenada en Plátano (Musa AAA Simmonds) cv. Gran Enano. Agrociencia (Cuba). 40(2):149-162.

17. PEÑA, E. 2005. Necesidades hídricas del banano y el plátano. 2005. [En línea] Disponible desde Internet en: http://www.revistas.mes.edu.cu/eduniv/02Libros-por-ISBN/959-16-0400/0349-Necesidades Hidricas_Banano_Platano.pdf (con acceso 04/12/10).

18. SANTACRUZ, G.; SANTACRUZ, E.E. 1995. Necesidades Hídricas del Cultivo del Banano en El Soconusco, Chiapas, México. Disponible desde Internet en: http:// www.plataneros.com.mx/archivos/NECESIDADES HIDRICAS_DEL_BANANO_EN_EL_SOCONUSCO_ CHIAPAS.doc (con acceso 07/12/11).

19. SASTRY, P.S.N. 1988. Agrometeorology of the banana crop. Agricultural Meteorology Report $\mathrm{N}^{\circ} 2$ 29. World Meteorological Organization (Italia). p.14-20.

20. TURNER, D.W.; FORTESCUE, A.J.; THOMAS, S.D. 2007. Environmental physiology of the bananas (Musa spp.). Braz. J. Plant Physiol. 19(4):463-484.

21. VAN VOSSELEN, A.; VERPLANCKE, H.; VAN RANST, E. 2005. Assessing water consumption of banana: traditional versus modeling approach. Agr. Water Managem. 74:201-218.

Recibido: Diciembre 6 de 2011

Aceptado: Julio 10 de 2012 